\title{
Supporting Information - Ultrafast All-Optical Switching in the Visible Spectrum with 6H Silicon Carbide
}

Long $\mathrm{Li}^{1,2, \#, \text { Xiao Guo }}{ }^{1, \#}$, Pengbo Ding ${ }^{1, \#}$, Zeyu Peng ${ }^{1, \#}$, Xihan Chen ${ }^{1}$, Yi Li ${ }^{3}$, Michael P.

Nielsen ${ }^{4,}$, Liang Guo ${ }^{1,5, *}$

${ }^{1}$ Department of Mechanical and Energy Engineering, Southern University of Science and Technology, Shenzhen, China

${ }^{2}$ School of Mechanical Engineering, Southeast University, Nanjing, China

${ }^{3}$ School of Microelectronics, MOE Engineering Research Center of Integrated Circuits for Next Generation Communications, Southern University of Science and Technology, Shenzhen 518055 , China

${ }^{4}$ School of Photovoltaic and Renewable Energy Engineering, University of New South Wales Sydney, NSW 2052, Australia

${ }^{5}$ Key Laboratory of Energy Conversion and Storage Technologies (Southern University of Science and Technology), Ministry of Education, Shenzhen 518055, China.

\# These authors equally contributed to this work.

*E-mail: michael.nielsen@unsw.edu.au; guol3@sustech.edu.cn

13 pages of supporting information, including 12 figures and 3 tables. 


\section{S1: The AFM topography image of the $6 \mathrm{H}-\mathrm{SiC}$ sample}

The atomic force microscope (AFM) is shown in Figure S1. The average roughness (the average distance of the datum from surface) is $0.218 \mathrm{~nm}$, which is dramatically smaller than the experimental light wavelength.

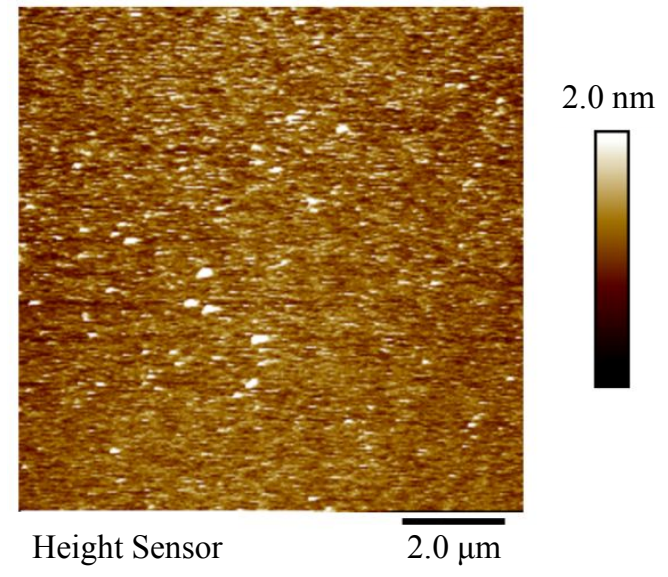

Figure S1. AFM topography of the $6 \mathrm{H}-\mathrm{SiC}$ sample. 


\section{S2: The XRD graph of $6 \mathrm{H}-\mathrm{SiC}$}

The X-ray diffraction (XRD) graph of $6 \mathrm{H}-\mathrm{SiC}$ is shown in Figure S2. There are two characteristic peaks, $\left(\begin{array}{lll}0 & 0 & 6\end{array}\right)$ and $\left(\begin{array}{lll}0 & 0 & 12\end{array}\right)$ at 35.6 degree and 75.3 degree, which indicate that the surface of the sample is perpendicular to the [0001] orientation.

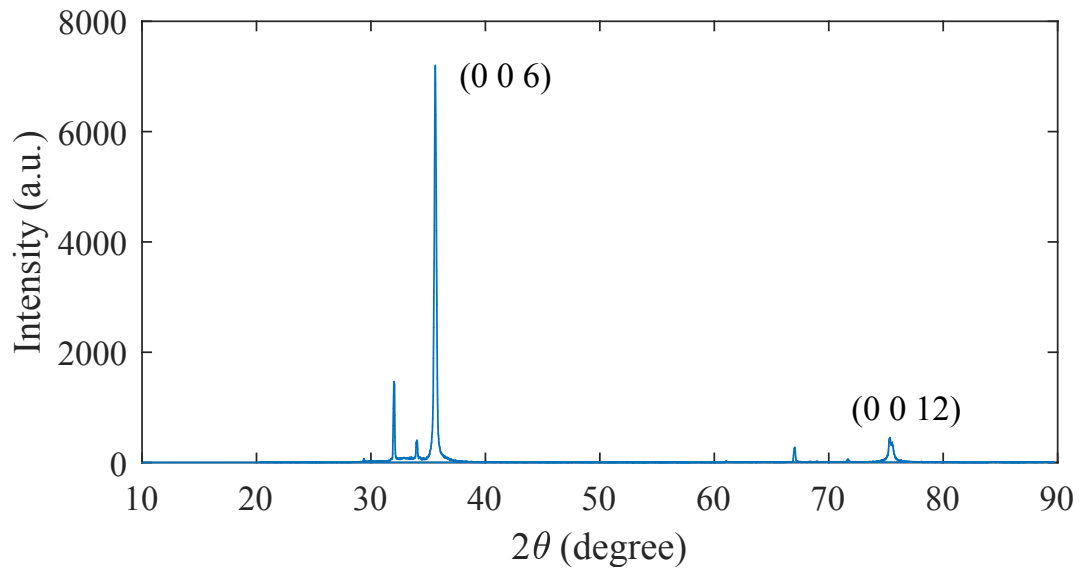

Figure S2. XRD graph of $6 \mathrm{H}-\mathrm{SiC}$. 


\section{S3: The linear absorption spectrum of $6 \mathrm{H}-\mathrm{SiC}$}

The linear absorption spectrum of the sample is shown in Figure S3. The linear absorption spectrum was measured by the UV/VIS/NIR spectrometer (Lambda $750 \mathrm{~S}$, PerkinElmer) at room temperature. The absence of absorption peaks above $430 \mathrm{~nm}$ demonstrates the low doping concentration of the sample.

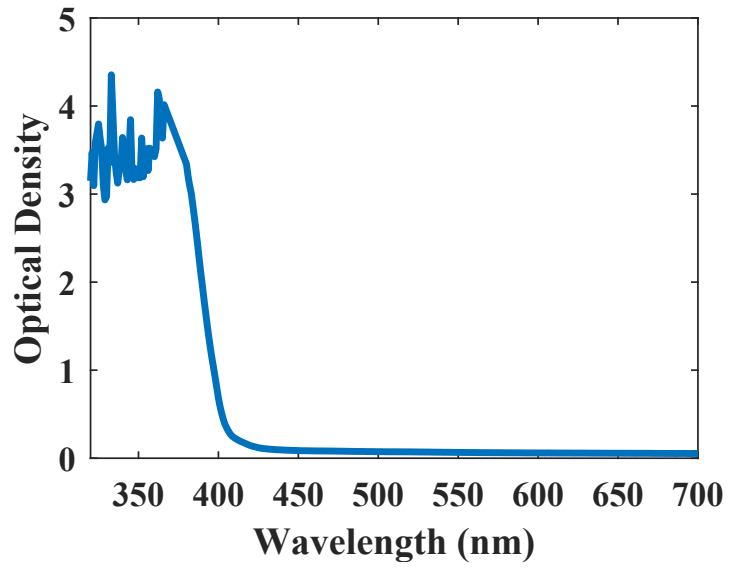

Figure S3. The linear absorption spectrum of $6 \mathrm{H}-\mathrm{SiC}$. 


\section{S4: The long decay time of the free carrier effect}

Figure $\mathrm{S} 4$ presents the signal for long decay time with probe wavelength at $430 \mathrm{~nm}$. An exponential decay $y=4.756 \times 10^{-4} \times \exp \left(-t /\left(5.063 \times 10^{-9}\right)\right)$ was obtained by fitting the experimental results from 10 ps to the end. Here y means the negative relative transmission change, and $t$ means the time delay. This means the long decay time of the free carrier effect could maintain as long as $5.063 \mathrm{~ns}$.

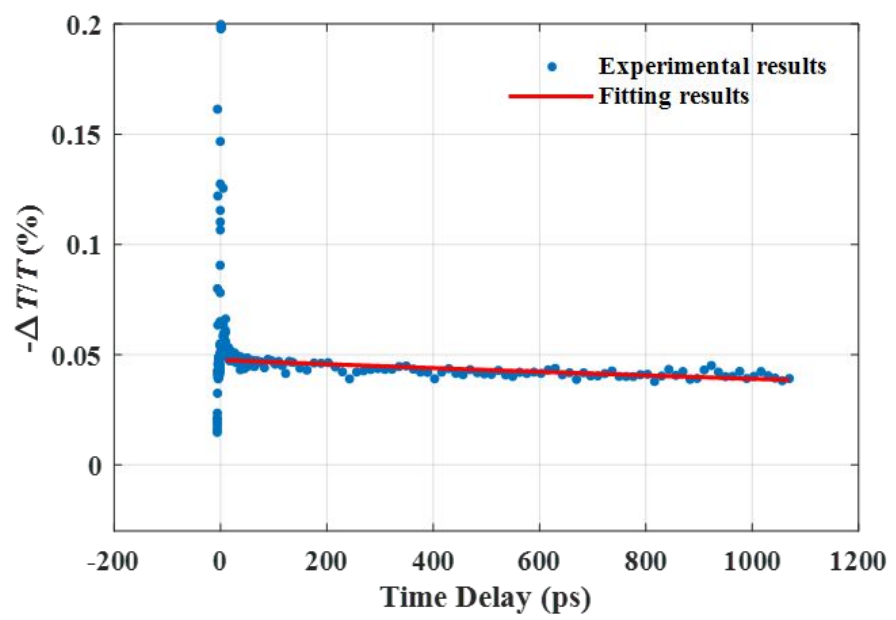

Figure S4. The signal for long time-delay with probe wavelength at $430 \mathrm{~nm}$. The pump wavelength was fixed at $820 \mathrm{~nm}$. The pump and the probe had the same polarization direction. 


\section{S5: The transient differential transmission signal for different probe wavelengths}

Figure S5 presents the transient differential transmission signals for different probe wavelengths. As there are three output bands for the OPA, the transient differential transmission signals of two probe wavelengths for each band are presented. In addition, the experimental and theoretical timedelay of the ghost peaks for different probe wavelengths were presented in Table S1. The experimental and the theoretical time delays of the ghost peaks (round trip times of the probe pulse within the sample) for different probe wavelengths are presented in Table S1. The experimental and the theoretical values show the same trend (The round trip time decreases as the probe wavelength increases), which proves that the weak peaks are induced by the reflection.

(a)

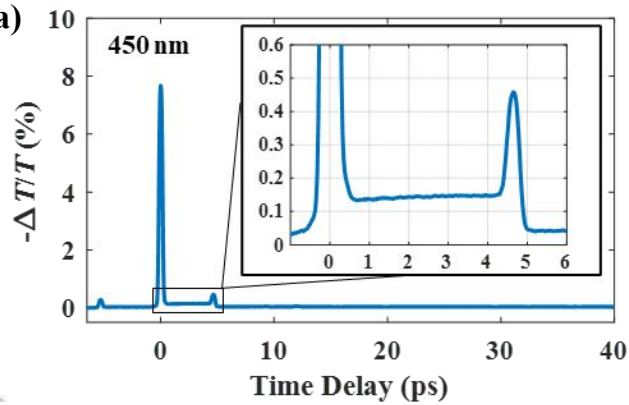

(c)

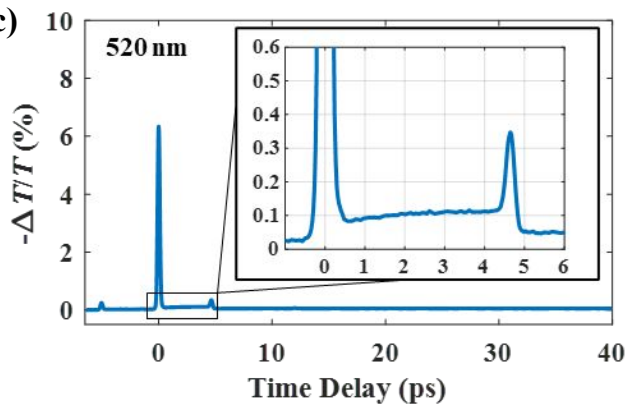

(e)

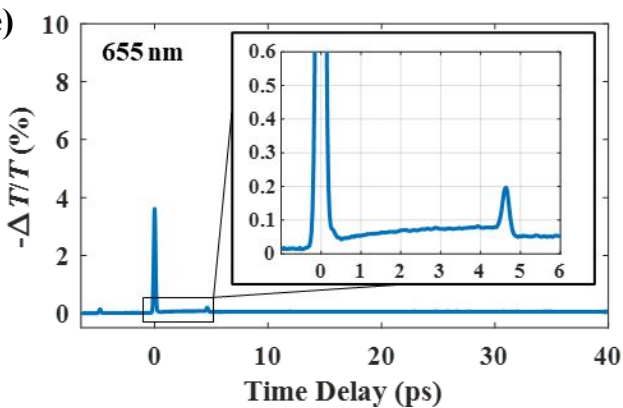

(b)

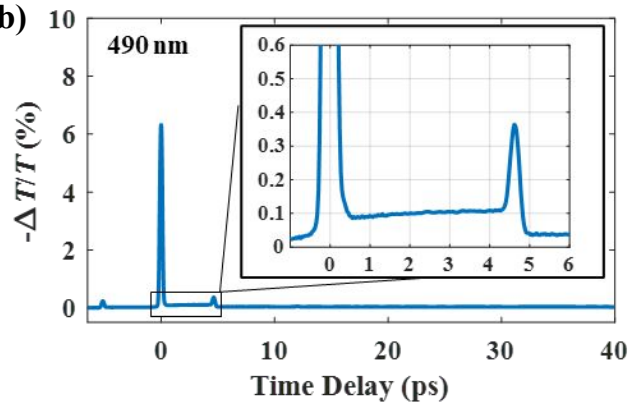

(d)

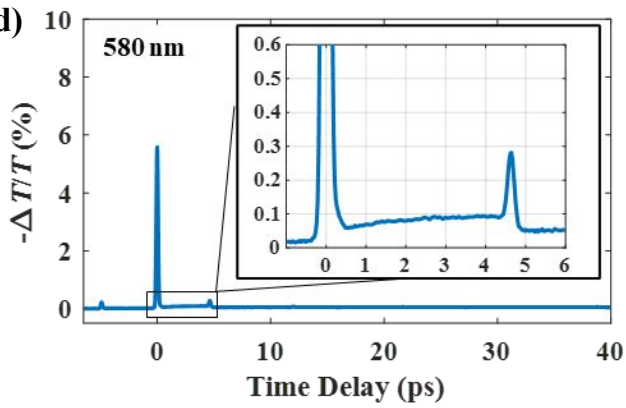

(f)

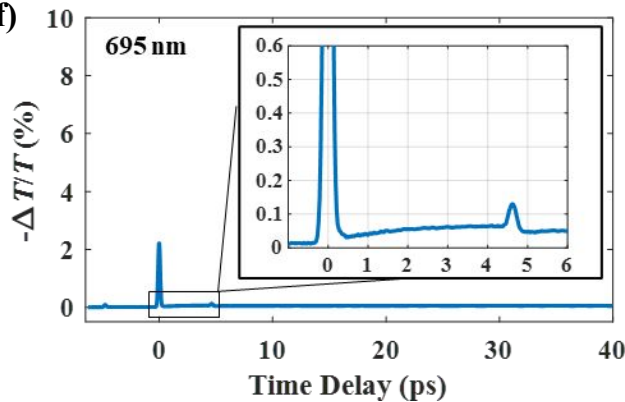

Figure S5. The transient differential transmission signals for different probe wavelengths. The pump wavelength was fixed at $820 \mathrm{~nm}$, and the fluence was fixed at $26.7 \mathrm{~J} / \mathrm{m}^{2}$. The pump and the probe had the same polarization. The probe was fixed at (a) $450 \mathrm{~nm}$, (b) $490 \mathrm{~nm}$, (c) $520 \mathrm{~nm}$, (d) $580 \mathrm{~nm}$, (e) $655 \mathrm{~nm}$, (f) $695 \mathrm{~nm}$. 
Table S1. The experimental and theoretical round trip time for different probe wavelengths.

\begin{tabular}{|c|c|c|c|}
\hline $\begin{array}{c}\text { Probe wavelength } \\
(\mathrm{nm})\end{array}$ & $\begin{array}{c}\text { The experimental round } \\
\text { trip time (ps) }\end{array}$ & $\begin{array}{c}\text { The refractive index } \\
\text { of 6H-SiC [S1] }\end{array}$ & $\begin{array}{c}\text { The theoretical } \\
\text { round trip time (ps) }\end{array}$ \\
\hline 450 & 5.28 & 2.722 & 4.68 \\
\hline 490 & 5.15 & 2.698 & 4.64 \\
\hline 520 & 5.04 & 2.685 & 4.62 \\
\hline 580 & 4.91 & 2.661 & 4.58 \\
\hline 655 & 4.80 & 2.635 & 4.53 \\
\hline 695 & 4.72 & 2.615 & 4.50 \\
\hline 820 & 4.64 & 2.598 & 4.47 \\
\hline
\end{tabular}

[S1] Choyke W. J.; Patrick L. Refractive index and low-frequency dielectric constant of 6H SiC. J. Opt. Soc. Am., 1968, 58(3): 377-379. 


\section{S6: The first pump propagation cycle inside the sample}

Figure S6a presents the schematic of the first pump propagation cycle inside the sample, which means the process that the pump first travels from the front surface to the rear surface, then is reflected by the rear surface, and finally travels back to the front surface. When the light path of the pump ahead of the probe is in the first pump propagation cycle, the transient differential transmission signal slightly increases due to the pump spatial propagation, as shown in Figure S6b. For two example time delays $t_{1}$ and $t_{2}$, the schematics of the pump propagation traces felt by the probe are presented in Figures S6c and S6d (The probe is spatially behind the pump by about $2 \Delta x$ ). In the first pump propagation cycle, the pump scanning region increases with the pump propagation, leaving more carriers along the scan trace. Therefore, as shown in Figures S6c and S6d, the overlap between the carrier-rich region and the probe scanning region increases with the time difference between the pump and the probe, and the effective absorption of the probe photons increases with the time delay. Thus, the signal slightly increased with the time delay from the main peak to the later weak peak, as shown in Figure S6b.
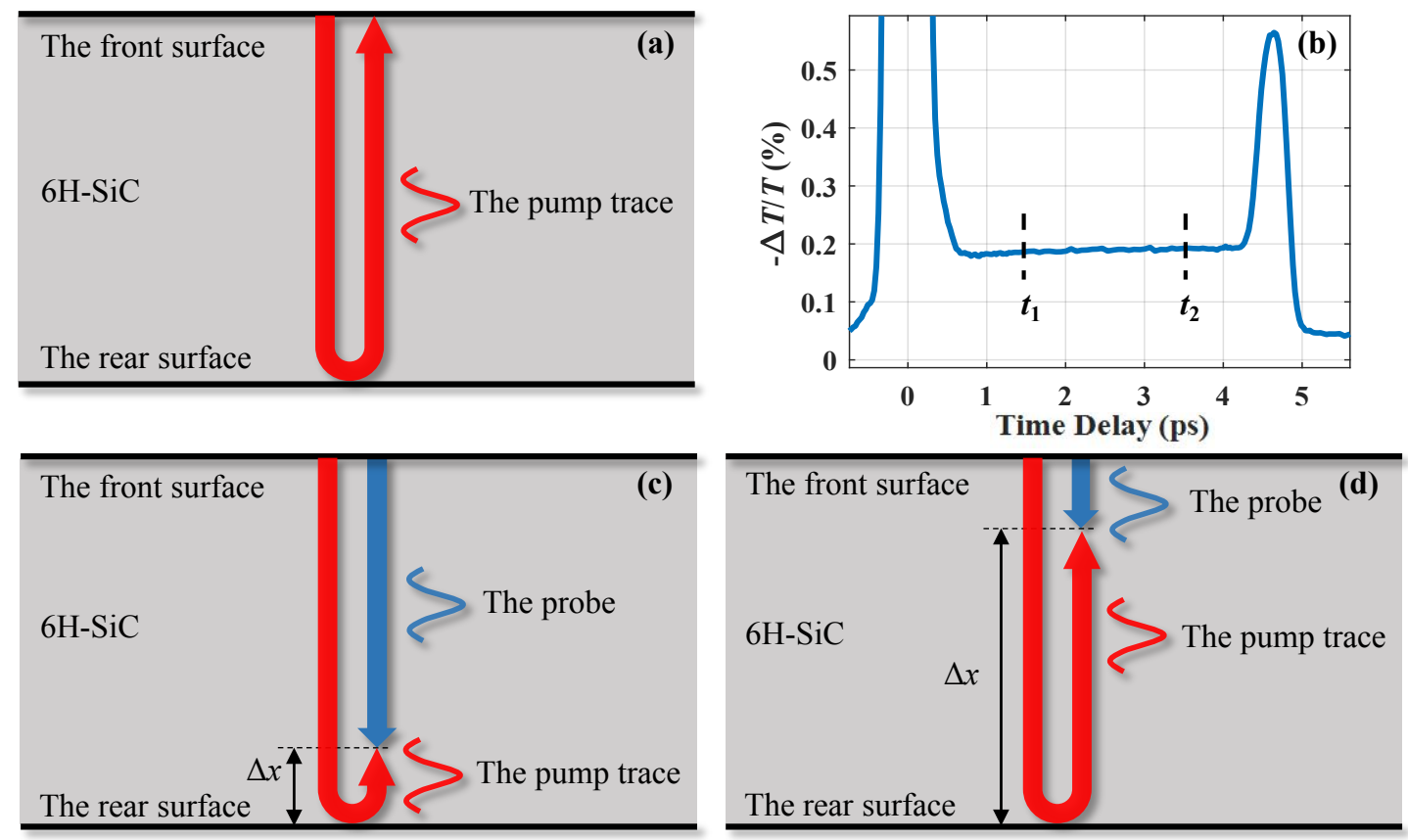

Figure S6. (a) The schematic of the first pump propagation cycle inside the sample. (b) The transient differential transmission signal from the main peak to the later weak peak. The schematics of the pump traces felt by the probe with time delays (c) $t_{1}$, (d) $t_{2}$. 
S7: The pulse widths and the spot sizes of the pump and the probe, and the calculated instrument response functions

The pump and the probe spot sizes and the pulse widths are necessary parameters in the numerical simulation and were measured in this work. They are given in Tables S2 and S3.

Table S2. The pulse width and the spot size of the pump at $820 \mathrm{~nm}$

\begin{tabular}{ccc}
\hline Pump wavelength $(\mathrm{nm})$ & Pulse width $(\mathrm{fs})$ & $1 / e^{2}$ spot diameter $(\mu \mathrm{m})$ \\
\hline 820 & 100.3 & 145.7 \\
\hline
\end{tabular}

Table S3. The pulse widths and the spot sizes of the probe at different wavelengths

\begin{tabular}{ccc}
\hline Probe wavelength $(\mathrm{nm})$ & Pulse width $(\mathrm{fs})$ & $1 / e^{2}$ spot diameter $(\mu \mathrm{m})$ \\
\hline 520 & 155.35 & 63.55 \\
540 & 138.55 & 34.64 \\
560 & 149.5 & 34.12 \\
580 & 140.1 & 42.24 \\
600 & 132.45 & 44.71 \\
620 & 132.9 & 44.64 \\
635 & 133.25 & 46.76 \\
635 & 121.95 & 35.79 \\
655 & 135.7 & 40.9 \\
675 & 158.9 & 45.11 \\
690 & 161.6 & 47.57 \\
700 & 160.25 & 48.45 \\
720 & 162.6 & 51.68 \\
\hline
\end{tabular}

The instrument response functions, the cross-correlation function of the pump and the probe, were calculated. The FWHMs of the instrument response function are $166 \mathrm{fs}$ and $188 \mathrm{fs}$ for the pump at $820 \mathrm{~nm}$, the probe at $600 \mathrm{~nm}$ and $675 \mathrm{~nm}$, respectively, as shown in Figure S7.
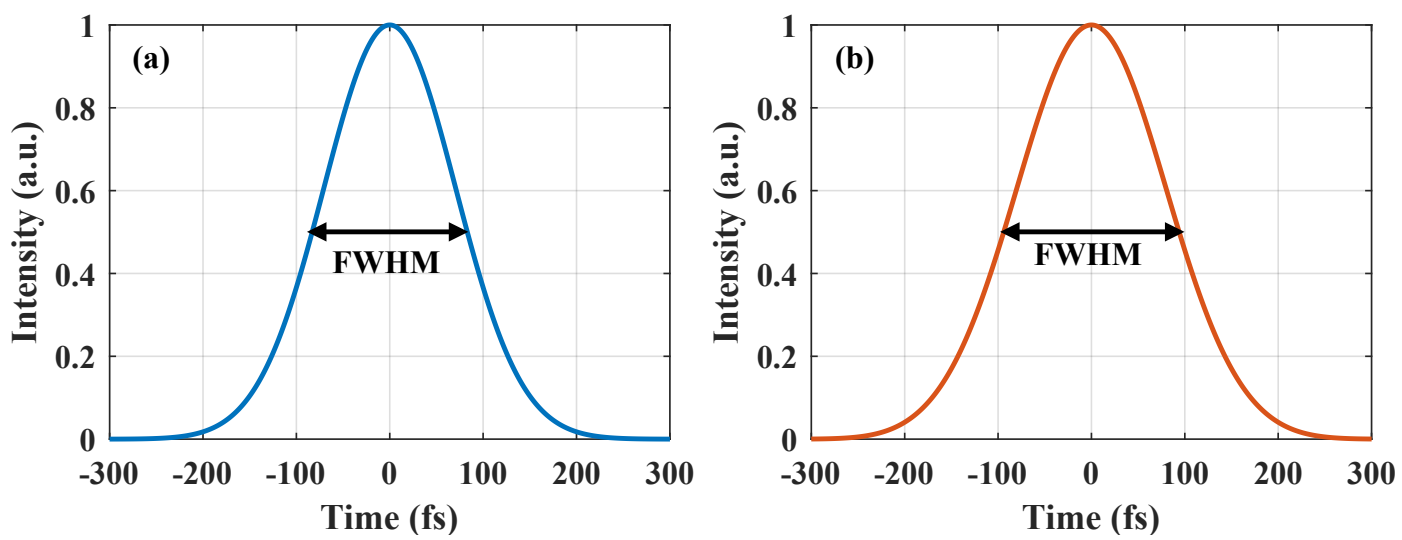

Figure S7. The instrument response functions for the pump at $820 \mathrm{~nm}$, the probe at (a) $600 \mathrm{~nm}$ and (b) $675 \mathrm{~nm}$. 
S8: The reflectance change induced by the nonlinear effect in the optical switching of the $6 \mathrm{H}-$ SiC.

The reflectance change induced by the nonlinear effect of the pump (the Kerr effect changes the real part of the refractive index and the nonlinear absorption changes the imaginary part)_was also considered in this work. Figure S8 depicts the calculated differential reflection due to the presence of the pump at $820 \mathrm{~nm}$ with fluence of $26.7 \mathrm{~J} / \mathrm{m}^{2}$ for different probe wavelengths, using the experimentally measured values for the nonlinear optical properties and pulse properties [S2]. The weak differential reflection on the order of $10^{-5}$, much smaller than the differential transmission on the order of $10^{-1}$, indicates that the experimentally observed transmission modulation is dominated by non-degenerate two-photon absorption.

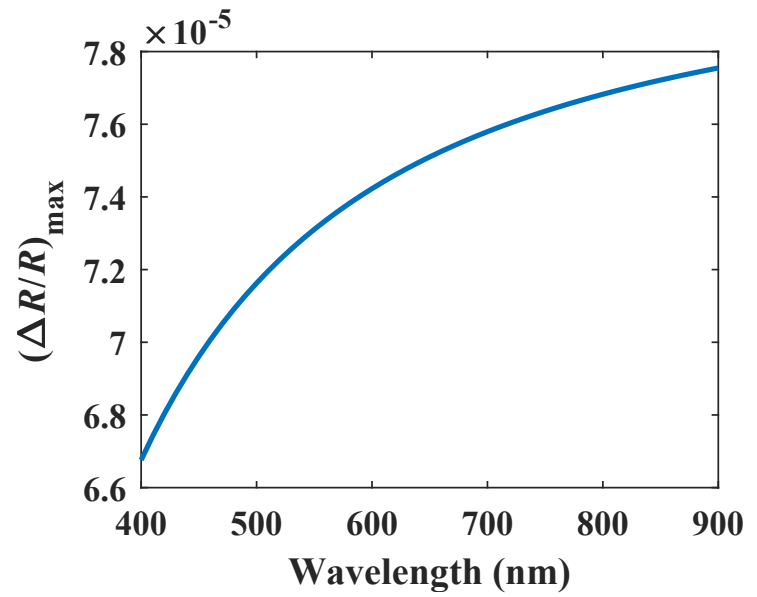

Figure S8. Calculated differential reflection induced by the presence of the pump for different probe wavelengths.

[S2] Guo, X.; Peng, Z.; Ding, P.; Li, L.; Chen, X.; Wei, H.; Tong, Z.; Guo, L. Nonlinear optical properties of $6 \mathrm{H}-\mathrm{SiC}$ and $4 \mathrm{H}-\mathrm{SiC}$ in an extensive spectral range. Opt. Mater. Express 2021, 11(4), 1080-1092. 


\section{S9: The dependence of $(-\Delta T / T)_{\max }$ on the probe fluence}

The differential transmissions for different probe fluences with wavelength $490 \mathrm{~nm}, 600 \mathrm{~nm}$, and $675 \mathrm{~nm}$ are given in Figure S9. In our experiments, the probe was fully received by a photodetector. Here, the output voltages of the photodetector were adopted to characterize the values of the probe fluence. The solid lines are the fitting curves of the experimental results, shown by the circles. The error bars are magnified by a factor of 5 . The horizontal fitting curves indicates that the probe fluence does not influence $(-\Delta T / T)_{\max }$, as in common pump-probe experiments.

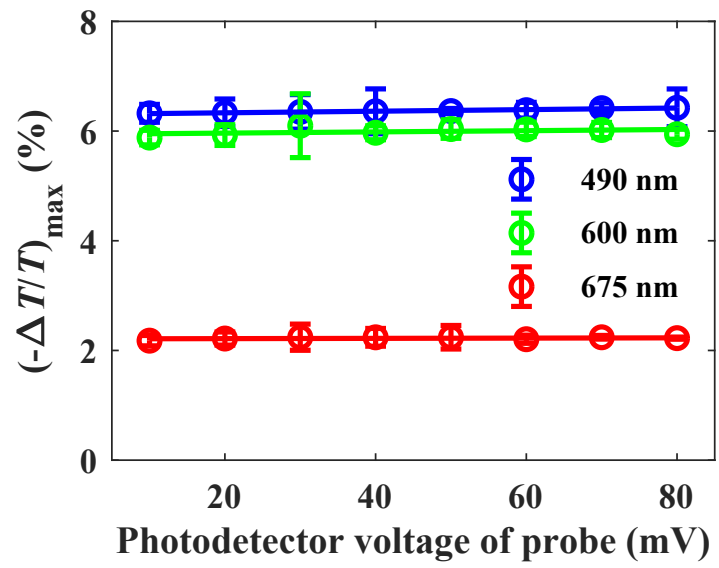

Figure S9. The dependence of $(-\Delta T / T)_{\max }$ on the probe fluence. The fluence of the pump was fixed at $26.67 \mathrm{~J} / \mathrm{m}^{2}$ with wavelength at $820 \mathrm{~nm}$. The pump and the probe had the same polarization direction. 


\section{S10: The dependence of $(-\Delta T / T)$ on the crystal orientation}

The influence of the crystal orientation on the modulation signal $(-\Delta T / T)$ was also examined in this work. Figure S10 shows the transient transmission signals at four random crystal orientations. The sample was rotated around the $c$-axis ([0001] orientation), which is perpendicular to the sample surface. There is no obvious difference among the signals, which clearly shows that the modulation signal( $-\Delta T / T)$ does not have relationship with the crystal orientation.

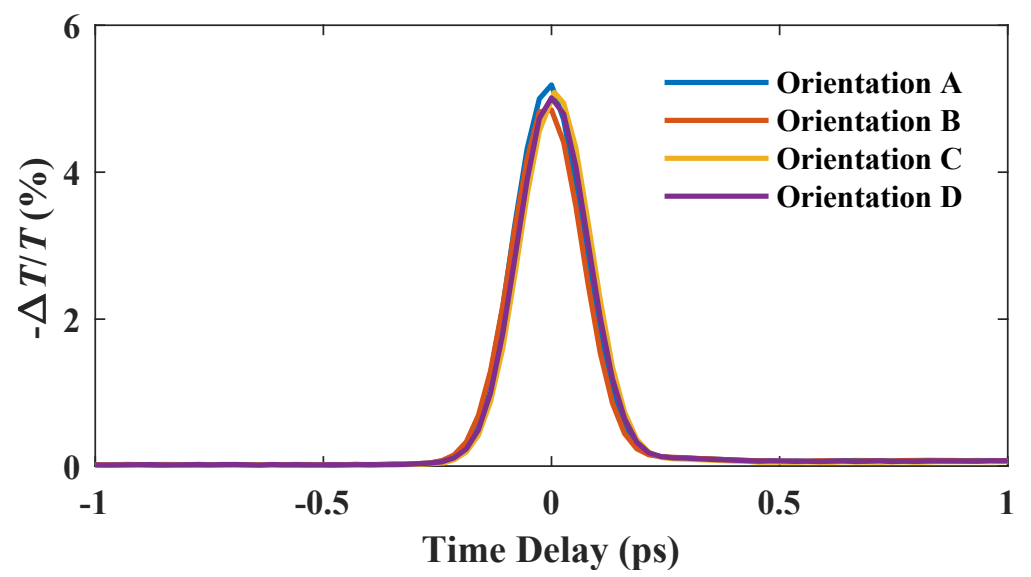

Figure S10. The evolution of $(-\Delta T / T)$ in different crystal orientations for the probe at $600 \mathrm{~nm}$ and the pump at $820 \mathrm{~nm}$ with a fluence of $26.67 \mathrm{~J} / \mathrm{m}^{2}$. The pump and the probe had the same polarization direction. 


\section{S11: The dependence of the FWHM on the pump fluence}

The FWHM values at different pump fluence for probe wavelength at $430 \mathrm{~nm}, 490 \mathrm{~nm}, 600 \mathrm{~nm}$, and $675 \mathrm{~nm}$ are presented in Figure S11. It indicates that the pump fluence does not affect the switching time significantly. The FWHM for probe wavelength at $430 \mathrm{~nm}$ show a marginal change at different pump fluence, which can be explained by the saturation phenomenon since the saturation effect tends to flatten the signal peak.

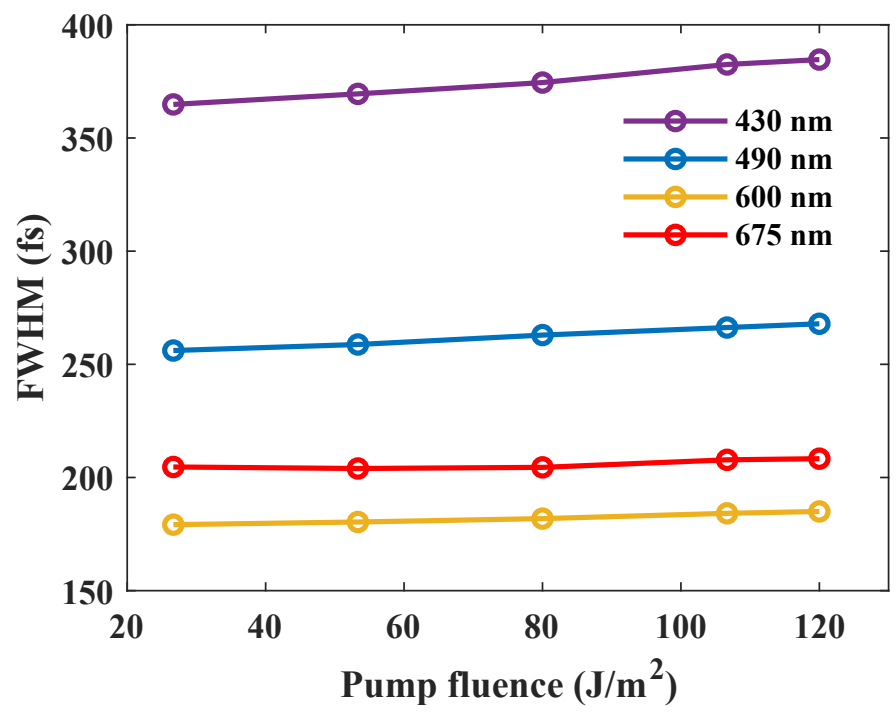

Figure S11. The FWHM of the signals for different pump fluences of probe at $430 \mathrm{~nm}, 490 \mathrm{~nm}$, $600 \mathrm{~nm}$, and $675 \mathrm{~nm}$. The pump wavelength was fixed at $820 \mathrm{~nm}$. The pump and the probe had the same polarization direction. 
S12: The dependence of $(-\Delta T / T)_{\max }$ on the pump fluence for long pump and probe wavelengths The dependence of $(-\Delta T / T)_{\max }$ on pump fluence at long pump and probe wavelengths is given in Figure S12. The solid lines are the quadratic fitting curves of the experimental results, shown as circles in Figure S12. The probe wavelength was set as $730 \mathrm{~nm}$, and the pump wavelength ranged from $820 \mathrm{~nm}$ to $920 \mathrm{~nm}$. In these cases, the sum of one pump and one probe photon energies was smaller than the band gap of $6 \mathrm{H}-\mathrm{SiC}$. The quadratic relationship between $(-\Delta T / T)_{\max }$ and the pump fluence indicates that the probe transmission change results from the absorption of two pump photons, which means that it is a non-degenerate three-photon absorption process.

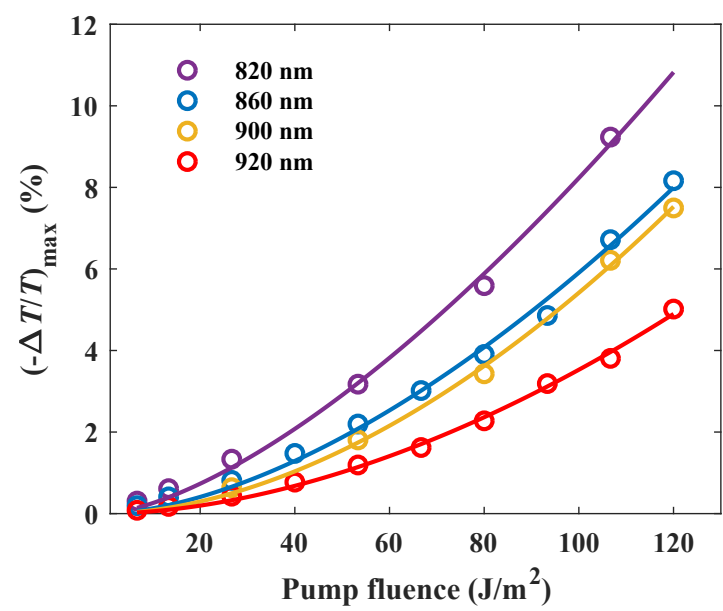

Figure S12. The dependence of $(-\Delta T / T)_{\max }$ on the pump fluence for long pump and probe wavelengths. The probe wavelength was fixed as $730 \mathrm{~nm}$. The pump wavelength ranged from 820 $\mathrm{nm}$ to $920 \mathrm{~nm}$. The pump and the probe had the same polarization direction. 